\title{
Tedious Versus Taxing: The Nature of Work in a Behavioral Health Context
}

\author{
MEGH MARATHE, School of Information, University of Michigan, USA \\ YOONSEON YI, School of Information, University of Michigan, USA \\ CHIA-HSUAN SU, School of Information, University of Michigan, USA \\ TING-WEI CHANG, School of Information, University of Michigan, USA \\ GABRIELA MARCU, School of Information, University of Michigan, USA
}

The goal of this study was to examine the work practices of behavioral health professionals with a view towards designing interactive systems to support their work. We conducted a qualitative workplace study, including in situ observations and semi-structured interviews, in a multidisciplinary clinic treating pediatric feeding disorders. This paper contributes a detailed characterization of clinicians' work practices and conducts a comparative analysis of three types of work: treatment, record management, and preparation work. We found that clinicians have a preference for taxing over tedious work. For example, they experience real-time data collection as more taxing but less tedious than retroactive data entry. Design efforts should balance the tension between addressing the taxing (data collection during meals) versus the tedious (manually entering data into spreadsheets). Although addressing the taxing improves within-routine efficiency, addressing the tedious improves overall morale. Further, we hypothesize that there is a rewarding or unrewarding quality to work that is dictated in part by its social, temporal, and clinical characteristics. We discuss conceptual and design implications for supporting clinical work, and highlight considerations unique to behavioral health.

CCS Concepts: • Human-centered computing $\rightarrow$ Empirical studies in HCI; Empirical studies in collaborative and social computing; • Applied computing $\rightarrow$ Health care information systems.

Additional Key Words and Phrases: clinical work; behavioral health; workplace studies; pediatric feeding

ACM Reference Format:

Megh Marathe, Yoonseon Yi, Chia-Hsuan Su, Ting-Wei Chang, and Gabriela Marcu. 2021. Tedious Versus Taxing: The Nature of Work in a Behavioral Health Context. Proc. ACM Hum.-Comput. Interact. 5, CSCW2, Article 302 (October 2021), 24 pages. https://doi.org/10.1145/3476043

\section{INTRODUCTION}

Clinical health informatics has long been concerned with how clinicians perceive and practice the different types of work demanded of them. The introduction and widespread adoption of electronic health records (EHRs) and order entry systems in hospitals has led clinicians to conceptualize their work into two non-overlapping categories: the work of taking care of patients (henceforth, "treatment work"), and the work of "caring for the chart," i.e., interfacing with EHRs and other digital systems (henceforth, we call this "record management work") [40, 50].

Authors' addresses: Megh Marathe, marathem@umich.edu, School of Information, University of Michigan, Ann Arbor, MI, USA; Yoonseon Yi, School of Information, University of Michigan, Ann Arbor, MI, USA, yiys@umich.edu; Chia-Hsuan Su, School of Information, University of Michigan, Ann Arbor, MI, USA, rexchsu@umich.edu; Ting-Wei Chang, School of Information, University of Michigan, Ann Arbor, MI, USA, changtw@umich.edu; Gabriela Marcu, School of Information, University of Michigan, Ann Arbor, MI, USA, gmarcu@umich.edu.

Permission to make digital or hard copies of all or part of this work for personal or classroom use is granted without fee provided that copies are not made or distributed for profit or commercial advantage and that copies bear this notice and the full citation on the first page. Copyrights for components of this work owned by others than the author(s) must be honored Abstracting with credit is permitted. To copy otherwise, or republish, to post on servers or to redistribute to lists, requires prior specific permission and/or a fee. Request permissions from permissions@acm.org.

(C) 2021 Copyright held by the owner/author(s). Publication rights licensed to ACM.

2573-0142/2021/10-ART302 \$15.00

https://doi.org/10.1145/3476043

Proc. ACM Hum.-Comput. Interact., Vol. 5, No. CSCW2, Article 302. Publication date: October 2021 
Researchers consistently find that clinicians enter the profession due to their investment in patient care, i.e., helping patients recover through treatment work. Instead, clinicians find themselves spending much of their time doing record management work such as navigating and updating EHRs, order entry systems, and various other information systems. Sun Young Park, Yunan Chen, and colleagues have shown that EHRs led to a four to five fold increase in documentation time in the emergency department $[4,36]$. Echoing these findings, scholars note that clinicians increasingly spend a majority of their day working on record management (e.g., writing a clinic visit note in the EHR after a patient encounter) $[1,3,4,9,40,46,50]$. Further, record management via EHRs places a heavier cognitive burden on clinicians because they have to remember the data for multiple patients instead of focusing on a single patient $[9,36]$. Clinicians see record management work as "clerical" and primarily administrative in nature. On the other hand, the patient-facing work of treatment is valued and considered the "real" work of medicine. Indeed, being forced into increasing amounts of record management work due to EHRs has been shown to increase the risk of physician burnout in the United States [50].

This scholarship tends to primarily focus on physicians, and behavioral health contexts have been understudied. We extend workplace studies scholarship to the realm of behavioral health by studying a feeding disorders clinic. We focus on technicians: paraprofessional clinicians who are responsible for implementing the treatment, typically under the supervision of a board-certified behavior analyst. Technicians are central to care provision in behavioral therapy, and so this paper complements the traditional workplace studies focus on physicians, nurses, and EHR [10].

We conducted a qualitative workplace study consisting of in-depth semi-structured interviews and observations at a Midwestern pediatric feeding disorder clinic. Behavioral therapy is an effective treatment for pediatric feeding disorders, but there are not enough professionals in this specialization to meet demand. Consequently, children with feeding disorders can wait for years to obtain access to treatment. Our motivation for studying the work practices within this context was to explore whether, and how, information systems could help support technicians' work, and thereby, scale available resources to give more children access to care.

Noting that treatment work was the most cognitively taxing while also most directly impactful to patient care, we initially proposed solutions to assist with the numerous tasks involved in treatment work. We shared sketches of high-level design concepts with feeding clinicians and expressly asked whether they would want information systems to support their work by, for example, reducing their multitasking, helping them stay focused on their patient, keeping their patients engaged, or supplementing their communication with patients and caregivers. Surprisingly, all clinicians said that they instead desired systems that would alleviate the tedious work of record management, which did not have immediate implications for patient care.

This paper parses clinicians' preference for performing taxing over tedious work. We draw upon two rich and divergent bodies of CSCW scholarship as explanatory frameworks to help understand the differential valuation of treatment and record management work in the feeding clinic. The first stream examines how different types of work are valued by studying their relative (in)visibility in a particular setting [56]. The second stream examines how clinicians experience different types of work by studying the temporal organization of work activities [44].

We find that clinicians experience treatment work as rewarding due in part to the high visibility, high social interaction, fast-paced action, and high clinical utility. Record management work, meanwhile, is slow, invisible, and perceived as serving an administrative purpose in this tightly-knit clinic where in-person updates are the norm. A third category, preparation work, is potentially tedious but not experienced as such because of its partial collaboration, immediacy, and necessity with indirect clinical utility. Hence, we hypothesize that there is a rewarding or unrewarding quality to the experience of work that is correlated with its social, temporal, and clinical characteristics.

Proc. ACM Hum.-Comput. Interact., Vol. 5, No. CSCW2, Article 302. Publication date: October 2021. 
This has both conceptual and design implications. Conceptually, we find that the visibility of work is intertwined with its temporal organization, and that how work is valued depends upon a combination of its perceived clinical utility and temporal organization in addition to its visibility. In terms of design, analyzing work in this way allows us to reconceptualize the analytical distinction between tedious and taxing work as a question of the intrinsic satisfaction that clinicians derive from some types of work. Thus, we should examine opportunities to reduce the burden of lowsatisfaction work, in addition to focusing on alleviating taxing work. Otherwise, well-intentioned attempts to support, streamline, or enhance clinical work may not result in adoption, uptake, or improved experience.

This paper makes the following contributions:

- Presenting a detailed characterization of the work practices of technicians in a behavioral feeding clinic, thus extending the workplace studies sub-field of CSCW research to the context of behavioral health. The paper also discusses how the problem space of behavioral health differs from commonly studied medical contexts.

- Describing a comparative analysis of three work practices that are common to most clinical settings-treatment, preparation, and health record management-along the lines of clinical utility, temporality, visibility, and experience of work.

- Theorizing that clinicians experience work differently depending upon a combination of its perceived clinical utility, visibility, and temporal urgency and pace. By doing so, the paper advances scholarship on the visibility and value of work in clinical settings.

- Demonstrating that certain forms of work can be rewarding despite being taxing while others can be tedious despite being straightforward, the paper raises conceptual and design implications for clinical information systems.

\section{BACKGROUND AND RELATED WORK}

\subsection{Workplace studies in healthcare contexts}

The workplace studies sub-field of CSCW research has long studied to what extent information systems could help scale existing clinical practice and improve access to care. However, such work has primarily focused on medical doctors, nurses, and the patient health record [10], only recently turning to examine behavioral therapy contexts (e.g., [30]). We extend this strand of workplace studies, contributing a detailed characterization of the work practices of behavioral health professionals in a pediatric feeding clinic. We draw upon the conceptual frameworks of temporality and invisible work to analyze how health professionals experience and value work.

2.1.1 Temporality in clinical work. Finding that problems of spatial organization and coordination tend to dominate CSCW literature, the early 2000s saw an emergence of scholarship focusing instead on the temporal organization and coordination of clinical work [5, 43, 44]. The conceptual framework proposed by Madhu Reddy and colleagues has proven particularly influential [44].

Drawing upon ethnographic fieldwork in a surgical intensive care unit and in conversation with Wanda Orlikowski and JoAnne Yates's practice-based organizational perspective [35], Reddy et al. propose studying the relationship between temporality and clinical practice, i.e., examining "temporality from the perspective of people doing the work and their interactions with temporal features of the work" [44]. They find that hospital work is organized and experienced in the form of three temporal structures: trajectories, rhythms, and horizons. Temporal trajectories are patientcentered: a temporal trajectory refers to the structured timeline of activities and circumstances that are created by a patient's illness trajectory. Temporal rhythms are collective in nature: these are recurring patterns of care and organizational work that occur on a daily basis, such as staff shifts. 
Temporal horizons are defined by an individual clinician: a temporal horizon determines how an individual organizes their current activities based upon a knowledge of anticipated future work. Clinicians routinely juggle multiple horizons, one per activity that they are responsible for. Temporal horizons can be flexible or inflexible: a horizon is inflexible if the activity must be completed by a certain time (e.g., EHRs must be updated before the shift changes), whereas a horizon is flexible if the activity completion deadline is looser or extensible. Further, horizons can be close or distant depending on when they are due: an activity that must be completed in the immediate future creates a close horizon (e.g., the 30 minutes before a shift ends); whereas one that is not due until much later has a distant horizon (e.g., a task due by the end of the fiscal year).

Reddy et al. find that within a particular unit, the temporal trajectories, rhythms, and horizons together determine how work is organized and experienced by clinicians. To borrow an example from Reddy et al., clinicians will often speed up their work in the 30 minutes before their shift ends so that they can go home on time and to ensure that clinicians on the next shift are sufficiently updated. This paper examines the relatively new context of behavioral health using Reddy et al.'s conceptual framework to understand how temporal trajectories, rhythms, and horizons structure work and impact clinician experience in the feeding clinic [44].

2.1.2 Visibility in clinical work. Noting that visibility plays an important role in determining the value and legitimacy assigned to work, Leigh Star and Anselm Strauss provide a framework to understand "how visibility and invisibility operate" in workplaces [56].

Star \& Strauss propose that work is rendered invisible due to three mechanisms: first, when employees themselves are invisible but the product of their work is visible. Such "non-person" employees are commonly seen in outsourcing or freelance contexts. Second, when employees are visible, but their work is relegated to the background. In clinical contexts, the "background work" of nurses, technicians, and other non-physicians is often invisible and undervalued compared to the work of physicians [51]. Third, when both employees and their work are invisible. For example, managers often use quantitative metrics of productivity as indirect substitutes for observable work in making decisions about resource allocations. Similarly, consumers of commoditized products such as smartphone users are kept unaware of the factory workers who manufacture the phones.

The visibility of work plays a key role in design considerations: in particular, "background work is vulnerable to oversight in the design of CSCW systems" [56]. However, Star \& Strauss stress that the solution is not as simple as providing detailed descriptions of background work and workers. Instead, visibility depends upon what counts as "real work," in any particular setting. Hence, it is important to first interrogate the meaning of work ("what is work?") in a specific site [56]. This paper uses Star and Strauss's framework to analyze the visibility and relative importance of different types of work carried out in the feeding clinic.

\subsection{The problem space of behavioral care}

We extend the literature by studying work practices in a behavioral health care context. Behavioral health is a multidisciplinary endeavor that applies psychological models of assessment and treatment to address physical and mental health concerns. Care teams consist of allied health professionals such as psychologists and speech-language pathologists working in collaboration with medical, nursing, and psychiatric providers.

Behavioral care delivery is complicated by social, psychological, environmental, and organizational factors [19]. Unlike some medical settings, behavioral care is oriented towards sustained interaction with patients and caregivers, in order to affect patient skills and behavior over time through interactive treatment sessions. Pediatric settings bring additional complexities in care 
goals, where treatment is dependent on facilitating the patient's autonomy while also helping caregivers understand how their own behavior will advance or hinder progress [2].

Further, behavioral health intersects in complex ways with disability. The multidisciplinary clinic we studied included applied behavior analysis (ABA) as one of its disciplines, and many patients were autistic children. When it comes to autism, attention-deficit/hyperactivity disorder, and similar disabilities, assessment and treatment can be based on subjective determinations of what is and is not appropriate social behavior [57]. The field of ABA has been the center of a firestorm of debate (e.g., $[7,53,54,59,61])$. A key criticism of ABA is that it has been used in attempts to change autistic children's behaviors to conform to social norms, rather than accepting them or targeting the social environment that discriminates against them [54, 60, 61]. Despite broad disagreement, nearly all clinics that treat people on the autism spectrum still have elements of ABA in their offerings.

Behavioral health services must therefore navigate the medical and social models of disabilityeither disabilities are viewed as deficits to be rectified through medical treatment, or disability is socially constructed via sociotechnical infrastructures such as unwritten norms for social behavior [49]. Disability studies scholars and activists have argued that services should be organized around a social model of disability, where disability is seen as a form of human variation that can become limiting in relation to a society dominated by neurotypical and non-disabled people [54].

\subsection{Pediatric feeding disorders}

The specific context we discuss in this paper is a clinic specializing in the treatment of pediatric feeding disorders. Feeding disorders occur in up to $10 \%$ of children without medical or developmental issues, $70-90 \%$ of children born prematurely and/or with chronic medical issues, and up to $90 \%$ of children with autism [20,21, 23, 26, 34, 47]. Children with feeding disorders have a traumatic relationship to food and exhibit aversion, selectivity, and trouble chewing or swallowing, causing gag reflexes and regurgitation due to underdeveloped oral-motor skills [20]. Untreated feeding disorders persist in 70-76\% of cases and have negative long-term impacts on mental health and social development, and in extreme cases, a failure to thrive [34].

In over $80 \%$ of cases, feeding disorders are thought to be behavioral in origin. That is, children learn to associate eating with pain or danger due to a complex interaction of medical, developmental, and environmental factors [21, 26, 34, 45]. Behavioral intervention based on applied behavior analysis (ABA) is the only evidence-based therapy proven to treat feeding disorders, but the demand far exceeds the capacities of the few existing trained professionals. Consequently, children currently wait over 20 months to be seen by treatment programs, and clinics have wait-lists of over 300 patients [34]. There is a need to investigate how information systems could help scale existing practice, improving access to treatment while maintaining efficacy and quality of care.

The limited HCI literature on pediatric feeding focuses on developing gamified interventions to improve self-feeding in children. Examples include a digitally-augmented plate and tableware system developed to promote vegetable eating in children [12,17]. Galoz et al. [11], Kadomura et al. [18], Leem et al. [24], and Lo et al. [27] meanwhile developed play-based interactive apps and sensing devices that motivate children to eat. This paper extends HCI literature on pediatric feeding to clinical contexts through a qualitative study in a pediatric feeding disorder clinic.

\subsection{Behavioral interventions in $\mathrm{HCl}$}

Treatment for pediatric feeding disorders utilizes rigorous behavioral data collection, the challenges of which have been a focus of research in HCI, across both clinical and classroom contexts. Perhaps most prominently, data collection has been studied in relation to autism-tools have been 
designed to reduce the burden of managing data through capture and access applications [13] and automated classification of behaviors [42]. These design efforts targeted managing large amounts of data in order to examine infrequent behaviors of interest, whereas our work focuses on the daily practices of data collection in clinical work. Demonstrating the potential of designing for this type of work, Kientz et al. [22] developed a well-received application supporting the structured data collection practices of therapists in the field of ABA. Prior work focused on behavioral health services for children has also analyzed the factors behind the persistence of paper for data collection [32], described the potential for computer-assisted data collection to support collaborative work [31], and explored technology for providing behavioral feedback directly to children [55].

Other HCI work in pediatric and adolescent care has focused on these patients' dependence on caregivers. Until young patients can be independent enough to communicate their preferences [25] and monitor how behaviors such as eating affect their health, so they can more effectively selfmanage their condition [29], they are dependent on others to do these things for them. Designing for the different roles that caregivers play can help them to coordinate care more effectively with clinicians, but caregivers can also partner with the patient in their care, and technology can help to facilitate a transition to more independence [33]. Hong and colleagues have shown how technology can enable pediatric patients and their families to explore and interpret complex clinical data such as radiology reports [15], and support the evolving partnership between patient and clinician as adolescents grow to become more involved in their care [16]. Similarly, Pina et al.'s work within the home has shown how family-based design can help children learn about self-monitoring their daily behaviors [38] and self-managing their health conditions [39].

\section{METHODS}

We conducted in-situ observations ( $n=23$ hours) and interviews $(n=5)$ with clinical staff members of a multidisciplinary pediatric feeding clinic housed in a large Midwestern teaching hospital. We focused on healthcare professionals' everyday work practices, hoping to find avenues for the design of information systems that would help clinicians and patients. This study was approved by the hospital's institutional review board. Participants provided written consent before the study began and verbal consent before each interview or observation.

We conducted five in-depth semi-structured interviews lasting an average of 45 minutes each with five out of the seven clinicians in the intensive feeding program: the psychologist \& director in charge of the program, two out of four behavior technicians (techs), the dietitian, and the speechlanguage pathologist. Interviews were audio-recorded and transcribed for analysis. Interviews focused on gathering information about clinicians' work, the use of paper in data collection, and understanding how clinicians experience the different kinds of work that form their daily routine.

We performed twenty three hours of observation over three months to gain a contextual understanding of clinicians' work practices. Observation sessions lasted between one and five hours each and we varied the time of day and day of the week when conducting observations to account for temporal differences. After an initial exploratory phase comprising seven hours, observations focused on two activities that shape the feeding clinic's workday: treatment sessions and health record management. During eleven sessions, we observed technicians treat four patients aged 1 to 12 years (we use 'child' and 'patient' interchangeably in the rest of the paper). Over five sessions, we observed technicians carry out record management duties such as transferring treatment session data to internal spreadsheets and patients' electronic health records. We also observed the time periods surrounding each session to contextualize it within the workday. Two members of the research team were present at and wrote field notes after each observation session. 
Clinic staff were primarily female (six out of seven), white (six out of seven), and their incomes ranged between $\$ 37,000$ (technician) and $\$ 92,000$ (psychologist). The majority female and white nature of our clinician participants reflects the demographic trends of the pediatric healthcare workforce in this hospital and in the United States [41]. Staff income was comparable to those of other psychological specialties in this hospital. Patients whose treatment sessions we observed were gender balanced and ethnically Black and white. Caregivers were primarily female (three out of four). Our research team was gender balanced, diverse in ethnicity and nationality, and familiar with this hospital through prior research, volunteering, and care experiences in other departments.

The research team built an affinity diagram to compare themes across data collection methods and sessions [14]. We examined all field notes and transcriptions and determined which observation and quotations should be included in the diagram. Selected information was transferred onto sticky notes. A total of 203 notes were clustered into groups with common themes in each category. We triangulated findings at the per-clinician and per-child level, wrote analytic memos, and conducted member checks with feeding clinic staff to ensure the validity of our findings [6, 8]. All names have been anonymized for participant confidentiality and quotes have been lightly edited for readability.

\section{CLINIC CONTEXT}

To characterize how technicians experience their work, we first provide an overview of the clinic context, its actors, and the nature of the treatment they provide. The feeding clinic we studied takes a multidisciplinary approach to the treatment of feeding disorders. The clinic offers two types of services depending on an initial assessment of the patient: an intensive treatment program and outpatient consultation. This paper focuses on the intensive treatment program. This program is staffed by seven full-time clinicians: a behavioral psychologist, a dietitian, a speech-language pathologist (SLP), and four technicians (techs). Upon intake into the program, children and caregivers meet with the psychologist and dietitian to formulate goals for treatment. The psychologist, dietitian, and SLP together develop a treatment protocol for the patient. The eight-week long intensive program requires children and caregivers to attend treatment five days per week, where the child is fed three meals per day by a tech to model and develop the correct oral-motor skills for eating. Feeding sessions are conducted in one of four treatment rooms.

We use the case of a patient named Jim to describe the salient actors in the feeding clinic: patients, caregivers, a behavioral psychologist, an SLP, a dietitian, and techs.

\subsection{Patients}

Jim is a 4-year-old boy with epilepsy and developmental delay resulting from a traumatic brain injury. He has been on a ketogenic diet to manage his seizures and he also has a history of gastroesophageal reflux disease. When he was one year old, Jim underwent a successful but traumatic swallow study (a study that lets clinicians examine the structure and function of the patient's throat and esophagus as the patient swallows liquids or solids coated with barium). Ever since that traumatic experience, Jim has refused to allow anything to enter his mouth. As a result, Jim's dietary requirements are being met entirely through a gastrostomy tube (a tube that delivers food directly to his stomach).

\subsection{Caregivers}

Jim's parents want him to be able to eat with his brother and friends in school, and to ideally become independent of the gastrostomy tube. Hence, Jim's primary care physician referred his parents to the feeding clinic. On contacting the clinic, Jim received an outpatient appointment for an initial screening evaluation. Pediatric patients must be accompanied by at least one caregiver during each clinic visit and Jim's mother accompanied him to the initial screening and all subsequent visits. 
Caregivers are asked to bring solid, pureed, and liquid foods that are particularly easy and difficult for the patient to eat and utensils or cups that the patient prefers using during meals.

\subsection{Behavioral psychologist and speech-language pathologist}

Working together, behavioral psychologist Anna and SLP Leslie conducted Jim's initial screening. They noted that Jim refused to open his mouth at first and gagged when the tip of his tongue was touched. In the rare instances where he permitted food to be placed on his tongue, he either did not swallow at all or swallowed at slower than expected pace. Indeed, Jim cried throughout his evaluation. Based on their assessment, Anna and Leslie decided that Jim would benefit most from the clinic's intensive program. They brought dietitian Mary into the room.

\subsection{Dietitian}

During initial screening meetings, the dietitian Mary seeks to understand the patient's and their family's typical dietary habits and propose a nutritionally adequate diet that meets treatment goals while also being sustainable in the context of the patient's family and/or school routines. Mary said, "a lot of my communication is usually more ... in terms of the home meal plans for our older kids, and talking to them a little bit about what ... are easier foods that they feel they can eat at school, with less pressure. So, I feel like a lot of my communication, in terms of behaviors, stem more around meal planning for our older kids." Leslie helps ensure that the texture of the food in the diet is safe with respect to the patient's current oral-motor skills and deficits.

In Jim's case, Mary recommended that the feeding clinic should focus on incorporating yogurt and apple into Jim's diet. Once Jim began the intensive program, Mary kept track of his daily food intake and made dietary adjustments along the way based upon the quantity and type of food he consumed. For example, once Jim started eating yogurt, the quantity of food ingested through his gastrostomy tube was reduced proportionally to account for the nutrition derived from the yogurt.

\subsection{Behavior technicians}

Psychologist Anna and pathologist Leslie constructed a treatment plan spread out over eight weeks for Jim. The plan consisted of the following steps: desensitizing Jim's gag response, teaching Jim to swallow applesauce and yogurt efficiently using a training spoon, switching Jim from the training spoon to a regular spoon, and increasing the volume of food Jim consumed orally. By the end of the program, clinicians want Jim to be able to eat one apple and a snack-sized container of yogurt. The treatment plan was open to change based on Jim's progress.

Jim's case was assigned to behavior technician (henceforth, "tech") Karen, who would be responsible for implementing Jim's treatment plan. The intensive program requires Jim and his caregiver to attend treatment five days per week, where Jim is fed three meals per day by Karen to model and develop the correct oral-motor skills for eating. Karen is involved throughout the intensive program: she is responsible for room and meal preparation before each treatment session; feeding, monitoring, and entertaining Jim during the session; and documenting outcomes after the session.

\section{CLINICAL AND NON-CLINICAL WORK}

In line with what has been reported in extensive literature on other contexts, we found that techs need to balance their clinical and non-clinical work, and this tension is central to their experience of the work. We therefore begin our description of their work practices by distinguishing these aspects. Techs are key to both day-to-day provision of care and to the overall functioning of the feeding clinic. Their work practices can be grouped into three broad categories: preparation, treatment, and 


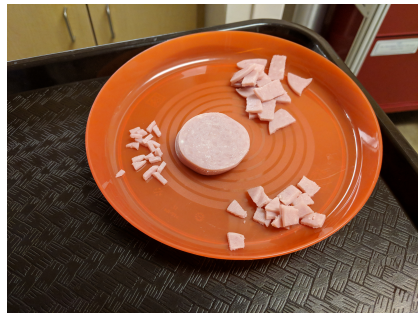

(a) Prepared food

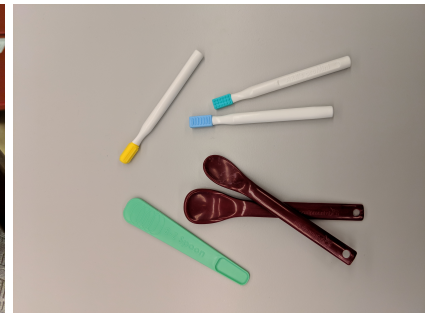

(b) Types of spoons

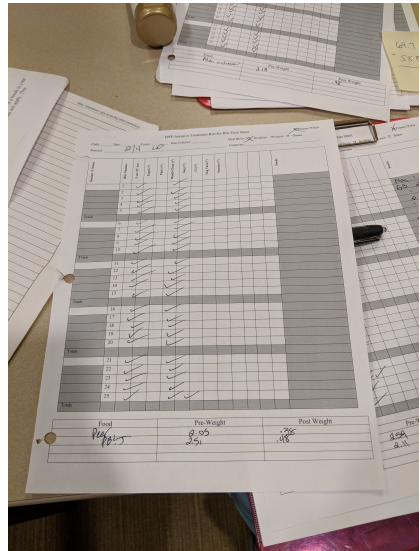

(c) Data sheet

Fig. 1. Common artifacts in the feeding clinic. Image (a) shows ham meticulously chopped into different portion sizes to train children to swallow larger bites. Image (b) shows the three types of spoons used to progressively develop oral-motor skills: three specialized brushes, a green flat spoon, and two purple regular spoons. Image (c) shows the paper data sheet used to record per-bite observations during treatment sessions.

\begin{tabular}{|c|c|c|c|}
\hline & Treatment & Record management & Preparation \\
\hline $\begin{array}{l}\text { Contribution to } \\
\text { patient care }\end{array}$ & $\begin{array}{l}\text { - Treat the patient } \\
\text { - Informally document } \\
\text { treatment and outcome } \\
\text { - Advance patient } \\
\text { through the illness } \\
\text { trajectory }\end{array}$ & $\begin{array}{l}\text { - Formally document } \\
\text { treatment and } \\
\text { outcome } \\
\text { - Document the illness } \\
\text { trajectory }\end{array}$ & $\begin{array}{l}\text { - Enable safe and } \\
\text { timely treatment }\end{array}$ \\
\hline $\begin{array}{l}\text { Visibility to patients } \\
\text { and caregivers }\end{array}$ & Highly visible & Invisible & Invisible \\
\hline Temporal rhythms & $\begin{array}{l}\text { Eight rhythms: } \\
\text { - Present food } \\
\text { - Check mouth } \\
\text { - Provide feedback } \\
\text { - Set break timer } \\
\text { - Monitor behavior } \\
\text { - Entertain } \\
\text { - Record data } \\
\text { - Prepare next bite }\end{array}$ & $\begin{array}{l}\text { Four rhythms: } \\
\text { - Digitize per-bite data } \\
\text { - Record quantity of } \\
\text { food consumed } \\
\text { - Aggregate data } \\
\text { for daily summary } \\
\text { - Enter summary } \\
\text { in EHR }\end{array}$ & $\begin{array}{l}\text { Four rhythms: } \\
\text { - Sanitize utensils } \\
\text { - Sanitize room } \\
\text { - Prepare meal } \\
\text { - Dispose leftovers }\end{array}$ \\
\hline Frequency & Thrice per day & Once a day & Thrice per day \\
\hline Duration & $\begin{array}{l}2.25 \text { hours } \\
\text { (45 min. per session) }\end{array}$ & $\begin{array}{l}1.5 \text { hours } \\
(30 \text { min. per session) }\end{array}$ & $\begin{array}{l}1.5 \text { hours }(20 \text { min. per } \\
\text { session }+30 \text { min.) }\end{array}$ \\
\hline Temporal horizons & Inflexible and close & Flexible and distant & Inflexible and close \\
\hline Function & Clinical & Administrative & Para-clinical \\
\hline Experience & Taxing & Tedious & Necessary \\
\hline
\end{tabular}

Table 1. Comparative analysis of techs' experience of treatment, record management, and preparation work. 
record management. Table 1 outlines our findings across these categories. We begin chronologically with preparation, and explain practices using the case of patient Jim and tech Karen.

\subsection{Preparation as para-clinical work}

Although not directly clinical in nature, preparation work is considered to serve an important para-clinical function in the feeding clinic without which patient care could not proceed. Techs spend approximately 1.5 hours of each day doing preparation work: roughly half an hour at the beginning of the day during which they sterilize meal utensils and trays to ensure ready availability for meals, and approximately twenty minutes before and after each treatment session.

Some preparation is tied to the collaborative work of the clinic, and will also have an immediate effect on how the treatment session goes with the patient, so the patient's needs are at the forefront. Before each treatment session, techs discuss the patient's treatment protocol with the psychologist, SLP, and dietitian to determine the feeding techniques and foods to be used. They prepare a meal tray containing precise portions of food and protocol-specific spoons (see figure 1 for examples) The treatment protocol specifies the type, quantity, and portion size of food items and the time at which new food items should be introduced. They print data collection sheets and write the pre-session weight of each food item onto the data sheet. They also sanitize eating utensils and rooms. The amount of careful work that goes into preparation is seen by techs as part of how they care for the individual needs of their patients. Tech Niki explained:

"One of the biggest contributing factors to feeding disorders is anxiety, especially with the older kids, and so having consistency and structure and predictability is really helpful for them. ... So we do things in a very systematic way and that's what makes us so successful. We have a specific size that we cut food up into. ... Then we'll do what we call texture feeding. So, instead of having two quarter by quarter inch pieces together on the spoon, we'll just make it one larger piece until we get to an age-appropriate portion because you don't want their parents at home cutting up quarter by quarter inch pieces."

Preparing meals, sterilizing utensils, and sanitizing treatment rooms helps ensure the safe, timely, and individualized delivery of treatment. Hence, preparation work is seen as para-clinical: necessary to the provision of care but one step removed from it.

\subsection{Treatment as centrally clinical work}

Treatment work is seen as central to patient care, because it directly helps the patient progress through the illness trajectory and advance towards the desired treatment goal. As part of treatment work, techs also do the important work of documenting fine-grained treatment decisions and outcomes. This documentation helps the multidisciplinary feeding team revise the treatment protocol as and when necessary. As a result, treatment work is seen as central to clinical work.

The tech typically begins a treatment session by spending 10 minutes desensitizing specific areas inside the patient's mouth using a specialized brush, with timed rest periods using a preset electronic break timer. Desensitizing areas and time intervals vary by patient. After desensitization, the tech proceeds with feeding the child while monitoring their behavior and recording data, until the meal timer goes off or the patient shows signs of fatigue. Techs do not aim to have patients finish the allotted food (although that would be a wonderful outcome). Instead, techs aim to have patients practice the correct oral-motor skills and minimize problem behaviors during meals. Hence, sessions in which patients show signs of fatigue are ended early. For instance, a tech said they ended a session because "when [patients are] fatigued, they're just practising the wrong oral-motor 
skills and we don't want that." If a session ends early, the tech manually turns the hand of the analog meal timer, with the resultant beep acting as an additional indicator of the end of the meal session.

Techs spend approximately 2.25 hours of each day doing treatment work, because sessions are conducted three times a day and each session typically requires 45 minutes with the patient. Thus, it comprises a significant portion of the tech's eight-hour work day.

\subsection{Record management as administrative and non-clinical work}

Techs spend approximately 1.5 hours of each day doing record management work. This consists of four sequential tasks: digitizing per-bite data, recording the quantity of food consumed, aggregating data for the daily summary, and entering the daily summary into the formal record. Ideally after each treatment session, or during the last working hour of the day, techs manually transfer the data recorded for the day's sessions from paper-based data sheets into the patient's electronic health record (EHR) and electronic behavior record (EBR). On average, techs take thirty minutes to type up and summarize the data for one treatment session into both electronic records. A significant portion of record management work therefore involves transforming data collected via the paper-based data sheet into formalized records in the patient's EBR and EHR. Yet in the tightly-knit feeding clinic, clinicians almost never look up information in the formal patient records systems (EBR and EHR), because they can easily ask one another.

The EHR is used mainly to comply with hospital requirements and for formal communication with caregivers and providers. In addition to the EHR, the feeding clinic maintains an informal repository of behavior data that we call the EBR. The EBR helps them record, maintain, and visualize information in a format customized to their needs [37]. The EBR contains fine-grained data that is specific to the feeding clinic, whereas the EHR contains high-level documentation for billing and administrative purposes. Unlike the EHR, the EBR is internal to the feeding clinic.

The EBR contains one Excel spreadsheet per patient that is updated daily during the course of the intensive program. The spreadsheet contains three tabs that are structured similarly to the paper-based data collection sheet. The first tab, titled Behavior Data, records the results of each five-bite trial and associated comments. The second tab, titled Ounce Data, records pre- and post-treatment food weights. The third tab, titled Daily Summary, displays daily summaries that are semi-automatically calculated based on the data entered in the previous two tabs. Techs manually transfer data from the paper-based data sheet to the spreadsheet. The goal is to generate graphs of the child's trajectory through the treatment program, but this currently remains unfulfilled because "it takes forever and a day" to generate graphs.

Record management is considered by clinicians to be non-useful work that is of the least relevance to care provision and work that takes time away from clinical work. Indeed, clinicians in the feeding clinic almost never refer to formal information systems (EBR and EHR) when, say, looking up the progress of a particular patient. Instead, clinicians just ask the relevant tech for a verbal update or for the paper-based data sheet from the patient's latest session.

One afternoon, tech Niki sat in the observation room of treatment room 1, watching tech Karen's treatment session with patient Jim. Tech Derek entered the observation room and sat down in front of a computer to eat lunch before typing up notes for his recent session with a different patient. A few minutes later, Niki called out to Derek saying, "can I see your data, Derek?" Derek handed over the paper-based data sheet from his treatment session. Niki looked at the data sheet for about five seconds, exclaimed, "cool," and handed the sheet back to Derek. There was no further discussion, but with a quick glance at the data sheet, Niki had obtained an update on Derek's patient. 
The importance of informal interaction carries over into clinician-caregiver interactions, where instead of asking caregivers to use the EHR, clinicians directly communicate results and clarify concerns in person. Tech Niki says, "typically, we'll just go in and talk to [caregivers] or show them the data [sheet] or sometimes I send emails just describing what I saw. If there's anything that I've been doing and I'm going to be out [of the office], I always just go in and talk to them about it. Right now, it's easy to do that because we're such a small program..." Anna added that daily summaries too are primarily delivered directly through verbal updates to caregivers: "we would go in and say, "wow, we had a really good day! He did great with the new foods except for peach but, he had a problem.... He got really upset for one bite and he was crying, and the second bite he got a little emotional, and the third bite he was talking to himself and he was happy. And that's how quickly the anxiety goes away."

Thus, the main function of record management work in this tightly-knit feeding clinic is seen as the creation of formal documentation of treatment decisions, outcomes, and trajectory for hospital administrative and billing purposes. Hence, clinicians consider record management work to be primarily administrative (and not clinical) work that does not directly contribute to patient care.

\section{TEMPORAL FEATURES AND THE EXPERIENCE OF WORK}

We describe the temporal features of preparation, treatment, and record management work to analyze how techs experience and value their work. As described earlier, Reddy et al. define three temporal structures-trajectories, rhythms, and horizons-which emerge from and dictate clinical work [44]. A temporal horizon defines how an individual organizes their work activities in response to temporal trajectories and rhythms and based upon anticipated future work. The temporal horizon for a task can be "close" or "distant" depending on how soon that task must be completed, and the horizon can be "flexible" or "inflexible" depending upon the flexibility of the task completion deadline. We find that the fast-paced, close, and inflexible horizons of treatment work lend it a sense of excitement and immediacy; whereas the distant and flexible horizons of record management work make it a tedious, slow, and fragmented experience. Although not fast-paced, preparation work has close and inflexible horizons to enable timely care delivery. Adopting a temporal lens thus helps us examine the connection between the experience and valuation of work.

\subsection{Preparation work as time-sensitive and inflexible}

Analyzing preparation work using the temporal structures framework, all four preparation tasks are time-sensitive and inflexible: sterilizing utensils in the morning; preparing meals before each session; sanitizing the treatment room and taking care of leftover meals after each session.

For instance, utensils and trays must be sterilized in the morning to ensure their ready availability during the rest of the day. Similarly, meals must be prepared according to the treatment protocol so that the session can begin on time and proceed as planned. The treatment room must be sanitized between sessions to prevent spreading infection between different sets of patients, caregivers, and clinicians. Finally, leftover food must be refrigerated, frozen, or disposed off soon after each session to comply with safety guidelines and to ensure that food reheated in future is safe for consumption.

\subsection{Treatment work as cyclic, fast-paced, and urgent}

The tech feeds the patient in a process that interweaves eight different work rhythms in a cyclic routine. These rhythms are:

(1) Present food - The tech presents precisely portioned bites of food to the patient using a protocol-specified spoon and carefully observes for feeding behaviors. Karen starts by feeding 
Jim pea-sized portions of applesauce on a green flat spoon. She holds the flat spoon like a pencil and rotates her wrist in a flipping motion to place the applesauce on Jim's tongue.

(2) Monitor behavior - The tech carefully monitors the patient's eating behavior to verify that they use the correct oral-motor skills and observe whether they exhibit any problem behaviors, such as gagging, expelling or retaining food in the mouth, negative vocalization, vomiting, and other child-specific problems. As Jim eats, Karen watches Jim and makes a mental note of problem behaviors. Techs also monitor the patient for health problems and new problem behaviors. For example, another patient with a tracheal tube once started coughing and gasping for breath between bites. The tech used the intercom system connecting the treatment room with the observation room, a seating area from the treatment session can be observed through a two-way mirror, to get the attention of SLP Leslie. The tech and Leslie re-adjusted the tube and the patient did not cough for the rest of the session. The intercom system is commonly used to communicate questions or concerns arising during the treatment session to observing clinicians, such as newly-observed and unusual patient behaviors.

(3) Check mouth - Five seconds after each bite, the tech examines the patient's mouth to ensure that they have swallowed the bite and their mouth is clean. This helps techs detect cases where patients push food to the sides of their mouth or under their tongue instead of swallowing it. After Jim appears to have eaten a bite of applesuace, for example, Karen says, "Fim, say aah?" Jim opens his mouth wide saying 'ah', and Karen finds no trace of applesauce in his mouth.

(4) Provide feedback - Noticing Jim's clean mouth, Karen smiles and says, "that was a super good bite!" After each bite, the tech provides feedback to the patient in the form of positive words, cheering and clapping. If the patient has not eaten, techs encourage them by saying for example, "eat at least one entire macaroni," and if the patient isn't using the desired oral-motor skills, "keep your tongue still, please."

(5) Set break timer - After providing feedback, techs use a preset digital break timer to reward the patient with a thirty-second-long break from eating (unless otherwise specified by the treatment protocol). Psychologist Anna explains that this break is intentionally designed to serve as a reward from the anxiety-provoking task of eating: "the 30-second break is the reward. The break is the main reward. So as soon as the kids take that bite and swallow it, they learn that we're going to leave them alone and not require them to eat or do the things that are hardest for them during that time. ... So they might as well just hurry and do it, get rid of the anxiety that they're having about it, and move on to something more pleasant [like playing]." Karen uses the break timer to reward Jim with a thirty second break. Depending on the patient, breaks can last from twenty seconds to one minute.

(6) Record data - During the short break, techs quickly record their observations from the previous bite by ticking several boxes on the paper-based data sheet (figure 1c shows an example data sheet). Tech Derek explains: "As soon as [the patient] accepts his bite, I press start on the timer, and it starts to count down. ... That's pretty much the only time there is to write it down. ... After each bite he gets a 30 second break and then I go and mark everything that I saw." Techs record several observations for each bite: was the bite successful (did the patient swallow this bite within five seconds resulting in a clean mouth)? Alternatively, did the patient exhibit one or more problem behaviors, such as gagging, expelling or retaining food in the mouth, negative vocalization, and other patient-specific problem behaviors? Answers are entered in the form of tick marks and occasionally a few scribbled words in the comments column. After Jim cleanly swallows a spoon of applesauce, for example, tech Karen checks off the boxes for five-second swallow and mouth clean.

(7) Prepare next bite - During the short break, the tech also prepares the next bite. In Jim's case, Karen prepares his next bite of applesauce on the flat spoon during the break. This is because 
with every beep of the break timer, the tech must move on to the next bite. If the patient has eaten well for $80 \%$ or more of five consecutive bites, the tech is to increase the bite size. If the child is eating multiple kinds of food, the tech also alternates between foods every few bites.

(8) Entertain - Throughout the session and in parallel with the other tasks, the tech is responsible for keeping the patient entertained by playing with toys, chatting about the patient's interests, playing competitive games on handheld devices, or playing cartoon videos on YouTube. For example, in one session, Jim played with a sterile glove that Karen had inflated and drawn a smiley face on. Karen also humored several questions asked by Jim, such as whether she resides in the hospital. Techs use toys and other forms of entertainment to include an element of comfort and play to treatment sessions. Psychologist Anna emphasizes that the use of entertainment in the feeding clinic is unlike the typical home context where entertainment is used as a reward for eating: "if kids start to get nervous and refuse [food], the adults try to make [eating] a game and offer their iPad." Instead, the clinic usually makes entertainment available regardless of the patient's eating performance: "Non-contingent reinforcement is something that [the patients] enjoy, it's just available all the time. So one of the kids, a seven year old, is on non-contingent reinforcement with tangibles - like he just gets to play the whole time, we don't take [the toys] away before the time." The form of entertainment also varies depending on the individual patient: "And there are some kids who have a lot of sensory deficits, kids who are blind and/or deaf or nonverbal and they don't have a lot of options for reinforcers. So if they like music, we'll just play music the whole session." Entertainment options include tangible toys such as stuffed animals, children's music, child-safe video games on handheld gaming consoles, and digital content such as YouTube videos on an iPad.

All eight tasks have close and inflexible horizons because of the flowchart-like nature of the tech's routine. For example, 'present food' has an inflexible horizon because the remaining tasks cannot proceed unless the food has been presented. Additionally, 'present food' has a close horizon because techs only have around 45 minutes within which to complete the 25 to 50 bites that patients are expected to eat in each session. Similarly, techs must monitor the patient's behavior immediately after presenting each bite, and techs must check the patient's mouth five seconds after they accepted the bite. Hence, 'monitor behavior' and 'check mouth' too have close and inflexible horizons. Finally, techs have less than thirty seconds within which to prepare the next bite, and hence this task too has a close and inflexible horizon.

\subsection{Record management as non-urgent and flexible}

Record management work consists of four tasks that are carried out in the following sequential order due to dependencies:

(1) Digitize per-bite data - Techs begin by filling out the first tab of the EBR spreadsheet (Behavior Data). This tab contains the same columns as the paper-based data sheet, but the value of each EBR cell is calculated by aggregating data from five consecutive cells from the paper-based data sheet. That is, one cell records the result of one "trial" of five bites. Each tick mark in the paper sheet counts as one, so cell values fall within the range [0-5]. The process of typing in cell values is fairly quick, taking 3-5 minutes overall.

Techs record additional data per bite depending on the patient and their progress. In the case of a patient like Jim who has difficulty swallowing food, for example, the tech recorded not only check marks indicating that a bite was successful but also the "latency time" or the amount of time required to swallow each bite. In other cases, techs may add comments indicating that they increased the bite size or switched to a new type of food at a particular point in the session. For example, a tech wrote "CN 2 peas" to indicate that she had increased the size of 
each bite of chicken nuggets from one to two peas. Such patient-specific measurements and comments require additional calculations and/or typing up handwritten comments. Hence, entering this additional data takes about 10 minutes, i.e., more than twice the time required to merely populate cell values based on check marks (as previously described).

(2) Record quantity of food consumed - The second tab of the EBR (Ounce Data) documents pre-session and post-session food weight in ounces. The tech types in the original food weights in the "pre" cell and the leftover food weights in the "post" cell. This triggers an automatic formula to calculate the value of the "intake (ounces)" cell. This is a very quick process, taking less than one minute on average.

(3) Aggregate data for daily summary - The third tab of the EBR (Daily Summary) generates a partially-automated daily summary based on the data entered in the previous two tabs. The daily summary reports the foods consumed, the average success rate (i.e., the percentage of bites during which the patient successfully swallowed the bite and had a clean mouth without exhibiting any problem behaviors), the current treatment protocol, and brief comments for each of the day's three treatment sessions (breakfast, lunch, and dinner).

The tech begins by manually calculating the average success rate for each session. To accomplish this, the tech must invoke the AVERAGE function in the correct cell and then drag her mouse cursor over the relevant cells to include them in the calculations. Techs often struggle with dragging their cursor over the designated cells, which results in an incorrect average. Techs are quick to notice such mistakes and reattempt calculation, but they find the manual parts of calculation frustrating. Psychologist Anna explains that techs consider record management as a whole and this part in particular to be extremely tedious for good reason: "for the techs, the tedious part is entering ... [the data] in Excel and trying to make the [summary] look right. I mean it's hours and hours, that's tedious. I mean, any time you have to take something off paper and put it into something else, you can setup the Excel Spreadsheet so that it grabs [the data] more or less automatically, but it doesn't usually work like that.... There's some errors somewhere in the coding and then the techs, you know it takes a long time [to troubleshoot]." Anna would like to use the EBR to automatically generate graphs of the patient's progress, but she doesn't currently do so: "right now, I don't ask to look at graphs because I know it's going to take the techs at least 30 minutes to prepare one. Whereas I know that I would ask to see them, and I know they would be running around excited to show them if we had them." Techs also type in brief comments summarizing each meal.

(4) Enter daily summary in EHR - After completing the EBR, the tech copies the daily summary and pastes it into a clinical progress note in the patient's EHR. In addition to the summary, the progress note contains boilerplate text that is auto-populated using other EHR data (e.g., patient name, age, gender, diagnosis, provider names) and fields with pre-populated template sections that need to be filled in by the tech. For example, the tech chooses between good, fair, and poor potential in the following sentence: "[Patient] demonstrates \{good/fair/poor\} potential for functional improvement due to [speech-language-factors for progress]." Similarly, the tech chooses between feeding and swallowing characteristics in this sentence: "[Patient] is a [age] [gender] with ${ }^{* * *}$ medical history who presents today with [diagnosis codes] characterized by \{feeding/swallowing characteristics\}." The completed progress note becomes part of the patient's EHR and is available to patients, caregivers, and other hospital staff.

All record management tasks are characterized by relatively flexible and distant temporal horizons. For example, recording per-bite data during treatment is a fast-paced and time-limited task with an inflexible and close temporal horizon. On the other hand, digitizing per-bite data is done offline (i.e., outside of treatment) and without any immediate task dependencies, thus leading to a flexible 
and distant temporal horizon. Similarly, there is no urgency to entering the daily summary into the patient's EHR, so long as it is completed before the end of the day.

\section{VISIBILITY AND THE VALUE OF WORK}

In addition to temporal features of their work, techs perform activities that vary in how visible they are to the other clinicians, their patients, and caregivers. The visibility of their work is also an important influence on how they value preparation, treatment, and record management work. We use Star and Strauss's conceptualization of invisible work to determine the perceived valued of work [56]. We find that treatment work is highly visible to all stakeholders and seen as the most legitimate form of work. In contrast, record management work is invisible and devalued because clinic stakeholders rarely use formal health records (preferring to exchange updates verbally and via data sheets). Notably, preparation work is similarly invisible to patients and caregivers, but it is vital to care delivery and occasionally serves as an opportunity for clinicians to socialize.

\subsection{Preparation work as mostly invisible but necessary}

We find that preparation work is mostly invisible to patients and caregivers. Tech Niki explains that this is because much of the preparation work takes place outside treatment sessions:

"Our first meal starts at 8:40. Once the dishes are done and everything is clean, then we will go in and check in with [psychologist Anna or SLP Leslie] about our plan for the day. [Dietitian] Mary will also tell us what foods we need to be focusing on, and then we'll get their trays ready. So we'll get a tray and go to the refrigerator and get any food that [the patient] needs. If we have food then it's really easy because we'll just grab it out of the fridge. Sometimes parents will bring in foods and so then we'll need to prepare them."

On rare occasions, preparation work becomes visible to other clinicians: techs with overlapping schedules congregate around the microwave as they prepare meal trays, exchanging jokes and pleasantries such as, "is that really chicken pizza? It smells like pot pie." Preparation work is thus mostly invisible but it is indispensable for safe and timely care delivery.

\subsection{Treatment work as highly visible and taxing}

During treatment sessions, techs' work is visible to all stakeholders. Active interaction with patients is an important part of every task that treatment work consists of. Further, although caregivers do not always understand the nuances of problem behaviors, oral-motor skills, or treatment, they are typically fully attentive throughout the session and trying to make sense of the treatment and its outcomes. We use Jim's second day at the clinic as an example.

During the lunch session, Jim's mom stayed in the observation room with tech Niki. Jim's mom carefully watches tech Karen feed Jim, and periodically asks Niki questions such as, "he closed [his lips]?” to clarify her understanding of Jim's eating behavior within the context of the treatment being provided. Later on in the session, Jim's mom also showed her awareness of the analog meal timer: while on the phone with a family member, Jim's glanced at the meal timer and said, "about five minutes left in this meal," to the caller without waiting for a confirmation from Niki.

The visible and attention-grabbing quality of treatment work is well-known to clinicians. Indeed, clinicians use this to their advantage, providing spur-of-the-moment knowledge in the form of "parent training" tidbits that parents and caregivers can use during meals at home. For example, tech Niki noticed that after Jim accepted a bite, he would immediately open his mouth for tech 
Karen to check (and praise) instead of first trying to swallow the bite. Using the intercom, Niki tells Karen to look away from Jim until she's ready to check his mouth. Niki explained to Jim's mom:

"So, he's trying to show right away and I think if we direct [Karen's] attention away and sort of, completely ignore him, I think we can get that [behavior] to go away, so then he'll swallow more quickly. ... He's not doing it to be bad or anything like that. He's just like, 'yay look at what I did!' I always, you know, whenever I do parent training, you can use your attention as a very powerful tool. What you pay attention to, they're going to do more of. What you ignore, they're going to do less of. So we're just using our attention here to shape up his behavior."

Treatment work is taxing for all stakeholders, but its high visibility and direct clinical utility ensure that it is seen as the most legitimate form of work in the feeding clinic.

\subsection{Record management as invisible and tedious}

The products of record management work are mostly invisible to patients and caregivers. Although record management results in the creation of daily progress notes in the EBR and EHR, these representations are difficult for patients and caregivers to interpret and make sense of given that the treatment trajectory spans the much longer period of eight weeks. Patients and caregivers mainly rely upon the immediacy of and derive interpretations based on their witnessing of treatment work. Psychologist Anna acknowledges this problem, saying that parents take visual evidence of gagging as a sign of treatment failure instead of paying attention to the nuances that are captured in the EBR, such as lowered intensity in gagging: "to the parents, their kid is gagging, [sometimes] multiple times on each trial. No matter how intense the gag is, they conclude, 'nothing is going well.' [If records were easily visible and accessible] you'd at least have something to show them."

Indeed, Anna and other clinicians in the feeding clinic wish that the products of record management could be made visible in the form of simple graphs or other representations that are easily comprehensible to both caregivers and techs. Anna said,

"[If we had visuals], we would look at [progress data] more. We'd be excited! Because the more you can see the kid's progress, [the more the treatment makes sense]. Sometimes, especially like the 12 year old [patient], the progress has been really slow. [SLP Leslie] and I can see [the progress] constantly, but it's harder for the techs and the parents to see.... Because [currently] they sort of just have to trust you, that you're doing this for a purpose and it's going to pay off, especially when it takes this long."

For techs, the visual products of record management could serve as an important indicator and motivator, showing that the treatment is helping. For caregivers, visuals could provide ways to make sense of how their child has progressed in the treatment.

However, visually representing the outputs of record management work remains neglected because record management work is already tedious and complicated. Pyschologist Anna explained:

"For the techs, the tedious part is entering, like taking the paper-and I was a tech too, that's where everybody starts in this field-so I've done all the paper data collection and then putting in Excel and trying to make the graph look right. I mean it's hours, hours, that's tedious."

Indeed, developing visual tools would require techs to dedicate additional time and energy to record management, a task that is already draining tech's time and motivation. Tech Niki details:

"it's the data collection and the graphing. That takes up a lot of time because right now we have to take paper data. You take the paper data, you put it into the graph. At the end 
of the day, then so we have this like daily summary tab where you go through when you average all the data from all the meals and then you take that data and you then put it into the medical record. But if you have any kind of change ... let's say in the middle of the day, you went from upright spoon to flip spoon. You'd have to average those separately. It's just like things like that. It's not hard, it's just time consuming, especially when we're trying to do dishes and flip over the rooms and all of those things. It can just be a lot."

That is, record management is seen as taking time away from para-clinical work (doing dishes and sanitizing rooms) and para-clinical work is seen as more relevant to care provision. Given the absence of both clinical utility and caregiver appreciation for record management, it remains seen as tedious, primarily administrative, and unrewarding work that is not relevant to patient care.

\section{DISCUSSION}

This paper examines work practices in a pediatric feeding clinic and describes features of work from the perspective of healthcare professionals, with consequences for the design of information systems. This research is among a growing body of work on behavioral health in HCI, but uniquely employs a workplace studies approach. In doing so, we also extend workplace studies scholarship beyond its traditional focus on physicians, nurses, and the EHR [10], and present a detailed characterization of the work practices of behavior techs. We first discuss the implications of a central tension in techs' experience of different types of work, and then discuss factors that distinguish the problem space of behavioral health from traditional medical care contexts.

\subsection{Tedious Versus Taxing Work}

We found a distinction between tedious and taxing work that in a broad sense mirrors the administrative and clinical dichotomy of work in healthcare.

8.1.1 Conceptual Implications. For techs, clinical work is rewarding despite being cognitively taxing, whereas administrative work is tedious despite in theory being important for the overall functioning of the clinic. Techs engage in three main types of work - treatment, record management, and preparation - that are valued in considerably different ways. Treatment work is fast-paced, action-oriented, patient-facing, social-interaction-driven, highly visible to patients, caregivers, and other clinicians, and perceived as being of the highest clinical utility. In contrast, record management is slow, fragmented, retroactive, computer-focused, mostly invisible, and perceived as serving primarily an administrative function. This negative conception is exacerbated by the current lack of visibility and clinical utility of formal records. As a result, record management is thought to draw techs' time, motivation, and energy away from the more legitimate work of patient care. Preparation work similarly involves little social interaction and is invisible to patients and caregivers. However, (unlike record management) preparation is considered important para-clinical work because it ensures the safe and timely delivery of treatment.

We hypothesize that there is a rewarding or unrewarding quality to the experience of work that is dictated in part by its social, temporal, and clinical characteristics. Prior work has theorized that the visibility of work determines what is valued as legitimate work in a particular setting [56]. We found that the relationship between visibility and value is more complex. In particular, we find that visibility is intertwined with the temporal experience [44] and perceived clinical contribution of work. The case of preparation work is noteworthy because its lack of visibility does not undermine its perceived legitimacy in the feeding clinic. In fact, clinicians were unwilling to spend more time in visualizing formal records due in part to their reluctance to take time away from preparation work. Thus, we extend conceptual understandings on the visibility of work, and foster dialogue between two streams of CSCW research on temporality and invisible work. 
8.1.2 Design Implications. The distinction between tedious and taxing work has implications for conceptualizing the work that individuals engage in and for the design of tools to support that work In the feeding clinic, the specific combination of high visibility, high social interaction, continuous fast-paced action, and high clinical utility together lends a rewarding quality to treatment work. In contrast, record management work, due to its combination of low visibility, lack of social interaction, slow repetitive tasks, and no perceived clinical utility, carried an unrewarding quality that techs did not appreciate. Preparation work seemed to achieve a nice balance, where there was no question about its clinical utility, even though it shared many characteristics with record management. We suggest that design efforts should also account for this rewarding quality or employee satisfaction for different kinds of work, and target efforts towards reducing the burden of low-satisfaction or less-rewarding work so that clinicians can spend more time on the rewarding type of work.

When coming up with ideas for interactive systems to support the feeding clinic, the designers on our team noted that real-time data collection during treatment sessions placed the highest cognitive burden on techs. Hence, designers directed their efforts at making real-time data collection less taxing for the tech, i.e., partially automating some of the numerous tasks that are interwoven in the fabric of treatment work. For instance, our team proposed having holographic butterflies and server robots to entertain the patient during meals, a wall-mounted sensor to automatically detect the patient's oral-motor or behavior problems, a smart desk to automatically detect food items and calculate pre- and post-treatment weights, and a smart watch that allows techs to record data via hand gestures instead of having to write on paper-based data sheets.

On presenting our designs to feeding clinic staff, however, we were univocally asked to prioritize alleviating the tedious work over assisting with taxing work. Hence, we suggest that design efforts should balance the tension between addressing the taxing (data collection during treatment) versus the tedious (record management work). This tradeoff is important because although addressing the taxing improves within-routine efficiency, addressing the tedious improves overall employee morale. In our specific case, the two happily coincide: streamlining real-time data collection could address both the taxing and tedious by reducing the amount of manual record management work at the expense of minor adjustments to treatment routines.

\subsection{Comparing Behavioral and Medical Care}

We confirm several findings from medical health informatics literature in the context of behavioral therapy. In particular, we show that behavior techs categorize their work in ways similar to medical practitioners: treatment work, i.e., the work of interactively treating the patient; and record management work, i.e., the work of "caring for the chart" [4,36, 40]. Further, techs too consider record management work as a tedious and clerical task that serves a primarily administrative goal. Indeed, the work of care in behavioral feeding operates through collaboration and labor hierarchies similar to those found in medical practice, where techs occupy the lowest-ranking position (in terms of salary and certifications) but are responsible for most of the bodily and interactional work with patients; whereas higher-ranking positions (e.g., psychologist) make overarching decisions about the treatment protocol and supervise the patient's trajectory from a slight distance.

However, important differences arose from our focus on behavioral health and on techs' work.

8.2.1 Preparation Work. Through the concept of preparation work, we add nuance to the polarizing stance of clinical versus administrative work that pervades discussions around the impact of EHRs on medical work. Although preparation work is also an important part of medical work, it is carried out by other members in the hospital's labor hierarchy, including volunteers. Physicians only concern themselves with treatment and record management work. In behavioral health contexts, the role of the tech is unique in that they also perform the additional category of preparation 
work, i.e., the work that goes into making treatment safe and timely. This category remains underdiscussed in existing scholarship focused on physicians. In our field site, preparation work is seen as para-clinical in that it helps make clinical work possible. As a result, preparation work is not viewed as a kind of drudgery, but instead as necessary work that must be performed for safe and effective care. Unlike medical contexts, that is, preparation work is intertwined with treatment in behavioral health. Importantly, preparation work is not the same as articulation work. Articulation work speaks to the coordination, allocation, and reallocation of tasks, resources, and personnel that makes work possible [48]. Preparation work, on the other hand, is a combination of routinized work (e.g., sanitizing rooms and utensils) and articulation work (e.g., making sure there is enough food on hand and that food is ready in time for an upcoming meal).

8.2.2 Sustained Interaction. Further, behavioral care is oriented towards sustained interaction with patients and caregivers, and teaching patients a set of skills through interactive treatment sessions, whereas the primary emphasis of medical training is on novelty in patient cases and learning through intervention. As a result, in behavioral care, play and entertainment are necessary and invaluable components of treatment work. This is in sharp contrast with the basic bedside manner and "detached concern" that are seen as important in medical care [58]. Additionally, in teaching hospitals such as the one we study, physicians are a largely migratory workforce of medical trainees undergoing apprenticeship (e.g., interns, residents, and fellows), whereas feeding clinicians follow career trajectories closer to those of nurses and techs (long-term positions in the same hospital).

8.2.3 Disability and Autonomy. Behavioral health services must also navigate the medical and social models of disability [49]. The clinic we studied included applied behavior analysis (ABA) as one of its disciplines, and many patients were autistic children. Scholars and activists have critiqued ABA for attempting to change disabled children's behaviors to conform to social norms [54, 60, 61] In the context of pediatric feeding disorders, the diagnosis on which clinicians primarily focus the treatment is not autism, but rather a feeding disorder that has medical (and not only social) consequences. However, there are aspects of this treatment that are more subject to conformity For instance, sometimes parents' treatment goals involve increasing the variety in their child's diet, not necessarily for nutritional purposes, but for the sake of social acceptance at school. CSCW research on behavioral health has begun to direct more attention toward goals of patient autonomy and self-management $[15,16,25,29,33,38,39]$, and we suggest more of this type of work with an explicit focus on the social model of disability and on promoting the autonomy of disabled children. For example, instead of Jim having to spend eight weeks in intensive treatment, what if Jim's friends learnt to accept his gastrostomy tube and perhaps assisted him in administering food through this tube? How could we enable Jim to participate in the design of his therapy?

8.2.4 Children Versus Caregivers as Recipients of Interventions. Finally, an important goal of pediatric behavioral health includes helping parents understand how their own behavior will or will not enable their child's progress [2]. Indeed, both in the meta reviews and in our own data (see example in section 6.3), we found that one of the main benefits of intensive feeding programs is "parent training," i.e., teaching parents and caregivers how to better care for and feed children [28, 52]. That is, the caregiver may actually be the one in need of intervention when a pediatric patient undergoes intensive feeding treatment. To rephrase, children receive a potentially stigmatizing diagnosis that is permanently entered into their medical record, when in fact it may be the caregivers who need diagnosis and training. In such cases, we suggest that healthcare and insurance infrastructures should be redesigned to avoid stigmatizing children by operating on caregiver medical records. 


\subsection{Limitations and Future Work}

This work has can be improved in several ways. We draw upon data collected in a pediatric feeding clinic in a large urban academic medical center. It would be interesting to compare our findings with studies conducted in independent, non-teaching, rural, and community-based clinics. Further, the experiences of patients and caregivers were outside the scope of the current study. Our future work will extend the analysis presented here to include the perspectives of non-clinician stakeholders This will be particularly critical for the design of tools that support clinicians' work, which will have impacts not only on clinicians. We propose a hypothesis about work satisfaction as a potential way to understand clinicians' preference for taxing versus tedious work. Alternative explanations may arise with more or different data, such as variation in clinicians' personal preferences. We plan to test this hypothesis through our continued engagement with the feeding clinic. Since the hypothesis seeks to explain a more general dichotomy between clinical and administrative work in healthcare, we will also seek opportunities to test its applicability in other contexts.

\section{CONCLUSION}

We conducted a qualitative workplace study to examine work practices in a multidisciplinary pediatric feeding clinic. Our study was focused on technicians, paraprofessional clinicians who are responsible for implementing the treatment, typically under the supervision of a board-certified behavior analyst. We characterized their work practices across three types of work: treatment, record management, and preparation work. Our analysis revealed a preference for taxing over tedious work. For example, they experience real-time data collection as more taxing but less tedious than retroactive data entry. We parsed this differential valuation of work by drawing upon explanatory frameworks on temporality and invisible work. Based on this analysis, we hypothesize that there is a rewarding or unrewarding quality to the experience of work that is correlated with its social, temporal, and clinical characteristics. We discussed the conceptual implications of this hypothesis, suggesting that the perceived value of work also depends upon its temporal organization and clinical utility. Therefore, we proposed that design efforts should balance the tension between addressing the taxing versus the tedious parts of work. Although addressing the taxing improves within-routine efficiency, addressing the tedious improves overall morale. Finally, we discussed considerations unique to behavioral health services such as fraught intersections with the social model of disability and the importance of caregiver training in fostering patient autonomy.

\section{ACKNOWLEDGMENTS}

We are grateful to the staff of the multidisciplinary clinic we observed in our fieldwork. We are indebted to Mark Ackerman, Nikola Banovic, Gillian Hayes, Os Keyes, Sun Young Park, Alanson Sample, and our anonymous reviewers for comments that helped improve the paper. We thank Amani Alkayyali, Michelle Chung, and Lucky Chowdhury for research assistance. This article is based upon work supported by the National Science Foundation under Grant No. IIS 1816319.

\section{REFERENCES}

[1] Joan S Ash, Dean F Sittig, Eric G Poon, Kenneth Guappone, Emily Campbell, and Richard H Dykstra. 2007. The extent and importance of unintended consequences related to computerized provider order entry. Fournal of the American Medical Informatics Association 14, 4 (2007), 415-423.

[2] Inbal Aviram, Naama Atzaba-Poria, Alison Pike, Gal Meiri, and Baruch Yerushalmi. 2014. Mealtime Dynamics in Child Feeding Disorder: The Role of Child Temperament, Parental Sense of Competence, and Paternal 
Involvement. Fournal of Pediatric Psychology 40, 1 (11 2014), 45-54. https://doi.org/10.1093/jpepsy/jsu095 arXiv:https://academic.oup.com/jpepsy/article-pdf/40/1/45/9727798/jsu095.pdf

[3] Lu Chen, Uta Guo, Lijo C. Illipparambil, Matt D. Netherton, Bhairavi Sheshadri, Eric Karu, Stephen J. Peterson, and Parag H. Mehta. 2016. Racing Against the Clock: Internal Medicine Residents' Time Spent On Electronic Health Records. Journal of graduate medical education (2016). https://doi.org/10.4300/JGME-D-15-00240.1

[4] Yunan Chen. 2010. Documenting Transitional Information in EMR. In Proceedings of the SIGCHI Conference on Human Factors in Computing Systems (CHI âĂŹ10). Association for Computing Machinery, New York, NY, USA, $1787 a ̂ A ̆ S ̧ 1796$. https://doi.org/10.1145/1753326.1753594

[5] Yunan Chen. 2011. Health information use in chronic care cycles. Proceedings of the ACM 2011 conference on Computer supported cooperative work - CSCW'11 (2011), 485-488. https://doi.org/10.1145/1958824.1958898

[6] J W Creswell. 2007. Qualitative Inquiry and Research Design: Choosing Among Five Approaches. SAGE Publications, Thousand Oaks, CA. https://books.google.com/books?id=DetLkgQeTJgC

[7] Elizabeth Devita-Raeburn and Spectrum. 2016. Is the Most Common Therapy for Autism Cruel? The Atlantic (2016).

[8] Robert M. Emerson, Rachel I. Fretz, and Linda L. Shaw. 2011. Writing ethnographic field notes.

[9] Oladimeji Farri, Karen A Monsen, Serguei V Pakhomov, David S Pieczkiewicz, Stuart M Speedie, and Genevieve B Melton. 2013. Effects of time constraints on clinicianâĂŞcomputer interaction: A study on information synthesis from EHR clinical notes. Journal of Biomedical Informatics 46, 6 (dec 2013), 1136-1144. https://doi.org/10.1016/j.jbi.2013.08.009

[10] Geraldine Fitzpatrick and Gunnar Ellingsen. 2013. A review of 25 years of CSCW research in healthcare: Contributions, challenges and future agendas. CSCW: An International fournal (2013). https://doi.org/10.1007/s10606-012-9168-0

[11] Ayelet Galoz, Orad Weisberg, Tal Kerencapelovitch, Yair Uziel, Ronit Slyper, Patrice L. (Tamar) Weiss, and Oren Zuckerman. 2014. ExciteTray. Proceedings of IDC (Jun 2014). https://doi.org/10.1145/2593968.2610476

[12] Sangita Ganesh, Paul Marshall, Yvonne Rogers, and Kenton Ohara. 2014. FoodWorks. Proceedings of NordiCHI 14 (Oct 2014), 147-156. https://doi.org/10.1145/2639189.2639225

[13] Gillian R. Hayes, Lamar M. Gardere, Gregory D. Abowd, and Khai N. Truong. 2008. CareLog: a selective archiving tool for behavior management in schools. In Proceedings of the SIGCHI Conference on Human Factors in Computing Systems. 685-694.

[14] Karen Holtzblatt, Jessamyn Burns Wendell, and Shelley Wood. 2005. Chapter 8 - Building an Affinity Diagram. In Interactive Technologies. 159-179. https://doi.org/10.1016/B978-012354051-5/50009-4

[15] Matthew K. Hong, Clayton Feustel, Meeshu Agnihotri, Max Silverman, Stephen F. Simoneaux, and Lauren Wilcox. 2017. Supporting families in reviewing and communicating about radiology imaging studies. In Proceedings of the 2017 CHI Conference on Human Factors in Computing Systems. 5245-5256.

[16] Matthew K. Hong, Lauren Wilcox, Daniel Machado, Thomas A. Olson, and Stephen F. Simoneaux. 2016. Care Partnerships: Toward Technology to Support Teens' Participation in Their Health Care. Association for Computing Machinery, New York, NY, USA, 5337âĂŞ5349. https://doi.org/10.1145/2858036.2858508

[17] Yeong Rae Joi, Beom Taek Jeong, Jin Hwang Kim, Joongsin Park, Juhee Cho, Eunju Seong, Byung-Chull Bae, and Jun Dong Cho. 2016. Interactive and Connected Tableware for Promoting Childrens Vegetable-Eating and Family Interaction. Proceedings of IDC (2016), 414-420. https://doi.org/10.1145/2930674.2930711

[18] Azusa Kadomura, Cheng-Yuan Li, Yen-Chang Chen, Hao-Hua Chu, Koji Tsukada, and Itiro Siio. 2013. Sensing fork and persuasive game for improving eating behavior. UbiComp Adjunct (2013). https://doi.org/10.1145/2494091.2494112

[19] Elizabeth Kaziunas, Michael S. Klinkman, and Mark S. Ackerman. 2019. Precarious Interventions: Designing for Ecologies of Care. Proceedings of the ACM on Human-Computer Interaction 3, CSCW (2019), 1-27.

[20] Jurgen Horst Kedesdy and Karen S. Budd. 1998. Childhood feeding disorders: biobehavioral assessment and intervention.

[21] M E Kerwin. 1999. Empirically supported treatments in pediatric psychology: severe feeding problems. JPP 24, 3 (1999). https://doi.org/10.1093/jpepsy/24.3.193

[22] Julie A. Kientz, Sebastian Boring, Gregory D. Abowd, and Gillian R. Hayes. 2005. Abaris: Evaluating automated capture applied to structured autism interventions. In International Conference on Ubiquitous Computing. Springer, 323-339.

[23] Tiffany Kodak and Cathleen C Piazza. 2008. Assessment and behavioral treatment of feeding and sleeping disorders in children with autism spectrum disorders. Child and adolescent psychiatric clinics of North America 17, 4 (2008), 887-905

[24] Sungmook Leem, Eun Jee Sung, Sungjin Lee, and Ilyoung Jin. 2018. MAMAS: Mealtime Assistance to Improve Eating Behavior of Children Using Magnetometer and Speech Recognition. In Proceedings of the 20th International ACM SIGACCESS Conference on Computers and Accessibility (ASSETS âĂŹ18). Association for Computing Machinery, New York, NY, USA, 483âĂȘ485. https://doi.org/10.1145/3234695.3240988

[25] Catherine Lim, Andrew B.L. Berry, Tad Hirsch, Andrea L. Hartzler, Edward H. Wagner, Evette Ludman, and James D. Ralston. 2016. " It just seems outside my health" How Patients with Chronic Conditions Perceive Communication Boundaries with Providers. In Proceedings of the 2016 ACM conference on designing interactive systems. 1172-1184. 
[26] Lene Lindberg, Gunilla Bohlin, and Berit Hagekull. 1991. Early feeding problems in a normal population. IfED 10, 4 (1991), 395-405.

[27] Jin-Ling Lo, Tung-Yun Lin, Hao-Hua Chu, Hsi-Chin Chou, Jen-Hao Chen, Jane Yung-Jen Hsu, and Polly Huang. 2007. Playful Tray: Adopting Ubicomp and Persuasive Techniques into Play-Based Occupational Therapy for Reducing Poor Eating Behavior in Young Children. UbiComp 2007 (2007), 38-55. https://doi.org/10.1007/978-3-540-74853-3_3

[28] Colleen Taylor Lukens and Alan H Silverman. 2014. Systematic Review of Psychological Interventions for Pediatric Feeding Problems. Journal of Pediatric Psychology 39, 8 (sep 2014), 903-917. https://doi.org/10.1093/jpepsy/jsu040

[29] Lena Mamykina, Arlene M. Smaldone, and Suzanne R. Bakken. 2015. Adopting the sensemaking perspective for chronic disease self-management. Fournal of Biomedical Informatics 56 (2015), 406-417.

[30] Gabriela Marcu, Anind K. Dey, Sara Kiesler, and Madhu Reddy. 2016. Time to Reflect: Supporting Health Services over Time by Focusing on Collaborative Reflection. In Proceedings of CSCW. ACM, New York, NY, USA, 954-964. https://doi.org/10.1145/2818048.2820079

[31] Gabriela Marcu and Allison N. Spiller. 2020. Collaborative Aspects of Collecting and Reflecting on Behavioral Data. In Proceedings of the 2020 CHI Conference on Human Factors in Computing Systems. 1-13.

[32] Gabriela Marcu, Kevin Tassini, Quintin Carlson, Jillian Goodwyn, Gabrielle Rivkin, Kevin J. Schaefer, Anind K. Dey, and Sara Kiesler. 2013. Why do they still use paper? Proceedings of CHI 13 (2013). https://doi.org/10.1145/2470654.2466436

[33] Andrew D. Miller, Sonali R. Mishra, Logan Kendall, Shefali Haldar, Ari H. Pollack, and Wanda Pratt. 2016. Partners in care: design considerations for caregivers and patients during a hospital stay. In Proceedings of the 19th ACM Conference on Computer-Supported Cooperative Work \& Social Computing. 756-769.

[34] Natalie Morris, R Knight, T Bruni, L Sayers, and A Drayton. 2017. Feeding Disorders. CAPC 26, 3 (2017), 571-586. https://doi.org/10.1016/j.chc.2017.02.011

[35] Wanda J. Orlikowski and JoAnne Yates. 2002. It's About Time: Temporal Structuring in Organizations. Organization Science 13, 6 (2002), 684-700. https://doi.org/10.1287/orsc.13.6.684.501 arXiv:https://doi.org/10.1287/orsc.13.6.684.501

[36] Sun Young Park, So Young Lee, and Yunan Chen. 2012. The effects of EMR deployment on doctors' work practices: A qualitative study in the emergency department of a teaching hospital. International fournal of Medical Informatics 81, 3 (2012), 204-217. https://doi.org/10.1016/j.ijmedinf.2011.12.001

[37] Sun Young Park, Katie Pine, and Yunan Chen. 2013. Local-universality: Designing EMR to Support Localized Informal Documentation Practices. In Proceedings of the 2013 Conference on Computer Supported Cooperative Work (CSCW '13) ACM, New York, NY, USA, 55-66. https://doi.org/10.1145/2441776.2441786

[38] Laura Pina, Sang-Wha Sien, Clarissa Song, Teresa M. Ward, James Fogarty, Sean A. Munson, and Julie A. Kientz. 2020 DreamCatcher: Exploring How Parents and School-Age Children Can Track and Review Sleep Information Together Proceedings of the ACM on Human-Computer Interaction 4, CSCW1 (2020), 1-25.

[39] Laura R. Pina, Sang-Wha Sien, Teresa Ward, Jason C. Yip, Sean A. Munson, James Fogarty, and Julie A. Kientz. 2017. From personal informatics to family informatics: Understanding family practices around health monitoring. In Proceedings of the 2017 ACM Conference on Computer Supported Cooperative Work and Social Computing. 2300-2315.

[40] Katie Pine. 2012. Fragmentation and choreography: caring for a patient and a chart during childbirth. In Proceedings of the ACM 2012 conference on Computer Supported Cooperative Work - CSCW '12. ACM Press, New York, New York, USA, 887. https://doi.org/10.1145/2145204.2145336

[41] Beth A Pletcher. 2020. The Pediatric Workforce: What to Expect. https:/www.aap.org/en-us/professional-resources/ Pediatrics-as-a-Profession/Pages/Private/The-Pediatric-Workforce-What-to-Expect.aspx

[42] Thomas Plötz, Nils Y. Hammerla, Agata Rozga, Andrea Reavis, Nathan Call, and Gregory D. Abowd. 2012. Automatic assessment of problem behavior in individuals with developmental disabilities. In proceedings of the 2012 ACM conference on ubiquitous computing. 391-400.

[43] Madhu Reddy and Paul Dourish. 2002. A Finger on the Pulse: Temporal Rhythms and Information Seeking in Medical Work. In Proceedings of the 2002 ACM Conference on Computer Supported Cooperative Work (CSCW'02). Association for Computing Machinery, New York, NY, USA, 344-353. https://doi.org/10.1145/587078.587126

[44] Madhu C Reddy, Paul Dourish, and Wanda Pratt. 2006. Temporality in medical work: Time also matters. In Computer Supported Cooperative Work, Vol. 15. 29-53. https://doi.org/10.1007/s10606-005-9010-z

[45] Kristi D Rivas, Cathleen C Piazza, Meeta R Patel, and Melanie H Bachmeyer. 2010. Spoon distance fading with and without escape extinction as treatment for food refusal. Journal of applied behavior analysis 43, 4 (2010), 673-683.

[46] Rubina Rizvi, Jenna Marquard, Gretchen Hultman, Terrence Adam, Kathleen Harder, and Genevieve Melton. 2017. Usability Evaluation of Electronic Health Record System around Clinical Notes UsageâĂŞAn Ethnographic Study. Applied Clinical Informatics 08, 04 (dec 2017), 1095-1105. https://doi.org/10.4338/ACI-2017-04-RA-0067

[47] Colin D Rudolph and Dana Thompson Link. 2002. Feeding disorders in infants and children. Pediatric Clinics 49, 1 (2002), 97-112.

[48] Kjeld Schmidt and Liam Bannon. 1992. Taking CSCW seriously. Computer Supported Cooperative Work (CSCW) 1, 1-2 (mar 1992), 7-40. https://doi.org/10.1007/BF00752449 
[49] Tom Shakespeare. 2010. The social model of disability. In The disability studies reader, Lennard J Davis (Ed.). Routledge, New York, NY, 266-273.

[50] Tait D Shanafelt, Lotte N Dyrbye, Christine Sinsky, Omar Hasan, Daniel Satele, Jeff Sloan, and Colin P West. 2016 Relationship between clerical burden and characteristics of the electronic environment with physician burnout and professional satisfaction. In Mayo Clinic Proceedings, Vol. 91. Elsevier, 836-848.

[51] Steven Shapin. 1989. The Invisible Technician. American Scientist 77, 6 (1989), 554-563. https://doi.org/10.2307/27856006

[52] William G. Sharp, Valerie M. Volkert, Lawrence Scahill, Courtney E. McCracken, and Barbara McElhanon. 2017. A Systematic Review and Meta-Analysis of Intensive Multidisciplinary Intervention for Pediatric Feeding Disorders: How Standard Is the Standard of Care? The fournal of Pediatrics 181 (feb 2017), 116-124.e4. https://doi.org/10.1016/j jpeds.2016.10.002

[53] Maxfield Sparrow. 2016. ABA-Unstrange Mind. http://unstrangemind.com/aba/

[54] Katta Spiel, Christopher Frauenberger, Os Keyes, and Geraldine Fitzpatrick. 2019. Agency of Autistic Children in Technology ResearchâĂŤA Critical Literature Review. ACM Transactions on Computer-Human Interaction 26, 6 (dec 2019), 1-40. https://doi.org/10.1145/3344919

[55] Allison N. Spiller, Karina Caro, Jonathan Arevalo Garay, and Gabriela Marcu. 2019. Supporting behavior management with a classroom display providing immediate feedback to students. In Proceedings of the 13th EAI International Conference on Pervasive Computing Technologies for Healthcare. 159-168.

[56] Susan Leigh Star and Anselm Strauss. 1999. Layers of Silence, Arenas of Voice: The Ecology of Visible and Invisible Work. Computer Supported Cooperative Work (1999). https://doi.org/10.1023/A:1008651105359

[57] Jason Turowetz and Douglas W. Maynard. 2019. Documenting diagnosis: testing, labelling, and the production of medical records in an autism clinic. Sociology of Health and Illness 41, 6 (2019), 1023-1039. https://doi.org/10.1111/14679566.12882

[58] Kelly Underman and Laura E. Hirshfield. 2016. Detached concern?: Emotional socialization in twenty-first century medical education. Social Science \& Medicine 160 (jul 2016), 94-101. https://doi.org/10.1016/j.socscimed.2016.05.027

[59] Daniel A. Wilkenfeld and Allison M. McCarthy. 2020. Ethical Concerns with Applied Behavior Analysis for Autism Spectrum "Disorder". Kennedy Institute of Ethics fournal 30, 1 (2020), 31-69. https://doi.org/10.1353/ken.2020.0000

[60] Rua M. Williams and LouAnne E. Boyd. 2019. Prefigurative Politics and Passionate Witnessing. In The 21st International ACM SIGACCESS Conference on Computers and Accessibility. ACM, New York, NY, USA, 262-266. https://doi.org/10. $1145 / 3308561.3355617$

[61] M. Remi Yergeau. 2018. Authoring autism: on rhetoric and neurological queerness. Vol. 33. $996-997$ pages. https: //doi.org/10.1080/09687599.2018.1457500

Received October 2020; revised January 2021; revised April 2021; accepted May 2021 